\title{
Modeling Effects of Bottom Curtain Weirs and Controlling Algal Blooms in Xiangxi Bay, Three Gorges Reservoir, China
}

\author{
Ranojit Kumar Dutta ${ }^{1,2,3, *}$, Jun Ma ${ }^{1,4}$, Baishakhi Das ${ }^{5}$, Defu Liu ${ }^{1,4}$ \\ ${ }^{1}$ College of Hydraulic \& Environmental Engineering, China Three Gorges University, Yichang, 443002, Hubei, China \\ ${ }^{2}$ Faculty of Science and Engineering, City University, Dhaka 1216, Bangladesh \\ ${ }^{3}$ Faculty of Engineering, Bangladesh University of Engineering and Technology (BUET), Dhaka-1000, Bangladesh \\ ${ }^{4}$ Hubei Key Laboratory of Ecological Restoration of River-lakes and Algal Utilization, Hubei University of Technology, \\ Wuhan 430068, China \\ ${ }^{5}$ International Relations, Department of Political Science, NU, Bangladesh \\ *Corresponding author: ranojit.dutta83@gmail.com
}

Received March 10, 2019; Revised April 23, 2019; Accepted May 23, 2019

\begin{abstract}
A bottom curtain weir (BCW) is a hydraulic structure that acts as a barrier to the flow and diffusion of heat across the width of a water body. Algal blooms occur frequently in the largest tributary of Xiangxi Bay (XXB) of the Three Gorges Reservoir (TGR). A laterally averaged two-dimensional hydrodynamic and water quality model was used to simulate BCWs, including water temperature, hydrodynamics and chlorophyll-a concentrations, for XXB. The numerical models show that BCWs are a much more attractive, much less expensive and time-saving algal bloom controlling technique for subtropical reservoirs. The developed model was calibrated using data collected in XXB from January to December 2010. The maximum chlorophyll-a concentrations observed were $125-154 \mathrm{mg} / \mathrm{m}^{3}$ according to sampling sites such as XX09, XX06 and XX01. Overall chlorophyll-a concentrations were markedly reduced by $4-44 \%$ as a function of BCWs height and location. A seasonal algal bloom reduction rate of more than 37\% was observed in summer. In some periods, such as May 27-31, June 2-4, June 16-18, August 16-18 and August 23-24, BCWs with heights of $3 \mathrm{~m}, 5 \mathrm{~m}$ and $7 \mathrm{~m}$ reduced algal blooms by up to 99\% at XX09 and XX06 in XXB. Therefore, the proposed BCWs can reduce algal blooms and improve water quality to save domestic water and aquatic ecosystems in XXB of TGR.
\end{abstract}

Keywords: Three Gorges Reservoir (TGR), Xiangxi Bay (XXB), algal blooms, CE-QUAL-W2, Bottom Curtain Weirs (BCWs)

Cite This Article: Ranojit Kumar Dutta, Jun Ma, Baishakhi Das, and Defu Liu, "Modeling Effects of Bottom Curtain Weirs and Controlling Algal Blooms in Xiangxi Bay, Three Gorges Reservoir, China." American Journal of Water Resources, vol. 7, no. 2 (2019): 50-57. doi: 10.12691/ajwr-7-2-2.

\section{Introduction}

Density currents occur frequently over the year in XXB of the Three Gorges Reservoir (TGR). A bottom curtain weir (BCW) is a hydraulic structure built across a river, channel or reservoir to divert flow $[1,2,3]$. Flow velocities in XXB have decreased dramatically since impoundment (2003) [2]. Algal blooms occur frequently in many tributaries, including XXB [2,4]. The economic benefits of TGR are contrasted with the negative impact on the aquatic environment, XXB is affected by density currents from upstream inflow, which is separated into overflow over the surface (warm water float) and underflow at the bottom (cold water underneath) [2]. Simultaneously intrusive density currents are entering XXB from the mainstream Yangtze River (YR) of TGR along the surface, middle and bottom as overflow, interflow and underflow
$[1,2,3]$. Density currents influence algal blooms, as these currents transport nutrients and trace elements $[2,5,6,7,8]$. There are a variety of conventional algal bloom control methods $[9,10]$. Some of these methods are not suitable for reservoir operation due to domestic consumption. Floating curtain weirs have been used to control algal blooms in reservoirs such as the Terauchi Dam Reservoir in Japan, Daceheong Reservoir in Korea, and So-oak River in Korea [9,11-14]. We previously studied floating curtain weirs (FCWs) for controlling algal blooms in XXB subtropical reservoir. FCWs divert overflow density currents, whereas internal curtain weirs (ICWs) affect interflow and control algal blooms easily without changing underflow. The algal blooms reduction rate may vary if underflows are interrupted by the application of BCWs in XXB.

Numerous investigations of algal blooms have been conducted for XXB, but previous studies have only partially discussed the exact reason and how to control 
algal blooms and the occurrence of bottom density currents. Given the deficiency of research on algal blooms and the occurrence of bottom density currents in XXB, we assessed a novel BCW method to divert bottom density currents and control algal blooms in XXB. BCWs have been used to control algal blooms in XXB through the CE-QUAL-W2 model. Here, we show the effects of BCWs in controlling algal blooms in the XXB subtropical reservoir. A $\mathrm{BCW}$ is an interior barrier that diverts the underflow density currents and diffusion of heat under the bottom of the water body. BCWs were installed at three different locations, upstream, midstream and downstream, corresponding to XX09, XX06 and XX01 of XXB and heights of $3 \mathrm{~m}, 5 \mathrm{~m}$ and $7 \mathrm{~m}$, respectively. A laterally averaged two-dimensional hydrodynamic and water quality model (CE-QUAL-W2) was used to simulate the BCW including water temperature, hydrodynamics and chlorophyll-a concentrations for XXB. The CE-QUALW2 model was calibrated using data collected in XXB from January to December 2010. The effects of the BCWs on hydrodynamics, water temperature, and algal blooms in the XXB subtropical reservoir were assessed. Numerical modeling of algal bloom control by BCWs is an attractive, less expensive and time-saving technique. Upstream cold inflow submerged to the bottom as an underflow simultaneously with intrusion of turbid intrusive underflow into the XXB from the mainstream Yangtze River of TGR [1,2,15]. Bottom slope density currents were significantly interrupted due to the effect of the BCWs. The upstream and midstream downslope bottom density currents were blocked by the BCWs simultaneously, and the mainstream YR intrusion water was barricaded by the downstream BCWs.

The objective of this paper was to control algal blooms in XXB of TGR by the application of BCWs through CE-QUAL-W2. We focused on the effects of the BCWs on hydrodynamics, thermal structure and chlorophyll-a concentration in XXB. The performance of the BCWs was evaluating based on the reduction of chlorophyll-a predicted by the model, termed the BCWs efficiency.

\section{Materials and Methods}

\subsection{Study Area}

$\mathrm{XXB}$ is located (from $110^{\circ} 25^{\prime} \mathrm{E}$ to $111^{\circ} 06^{\prime \prime} \mathrm{E}$ and from $30^{\circ} 57^{\prime} \mathrm{N}$ to $31^{\circ} 34^{\prime} \mathrm{N}$ ) at the lower reach of TGR and the middle reach of the YR in Hubei Province, China (Figure 1a). XXB is the largest tributary of the TGR. Downstream of XXB connects with the mainstream YR. Large amounts of dense currents intrude into XXB at different water levels (Figure 1(c)) [2].

\subsection{Incorporation of Bottom Curtain Weirs with the CE-QUAL-W2 Model for XXB}

CE-QUAL-W2 is a 2D hydrodynamic and water quality model $[16,17]$. The model grid discretizes a waterbody into computational cells whose locations are defined by their segment $[\mathrm{I}]$ and layer number $[\mathrm{K}]$, i.e., cell $(\mathrm{K}, \mathrm{I})$ in Figure 1 (b). The BCWs were set at specified cell locations such as segment and layer number. The BCWs were always in the reservoir bottom during the simulation period (Figure 1(c)). BCWs were on the downstream side of a segment. Here, BCWs are set at the bottom of the reservoir with heights of $3 \mathrm{~m}, 5 \mathrm{~m}$ and $7 \mathrm{~m}$ respectively at XX09, XX06 and XX01, respectively (Figure 1(b)). All meteorological data were obtained from a hydrological station at Xingshan shown in Figure 1 (a) and the China Three Gorges Corporation (CTGC) for the year 2010 [2].
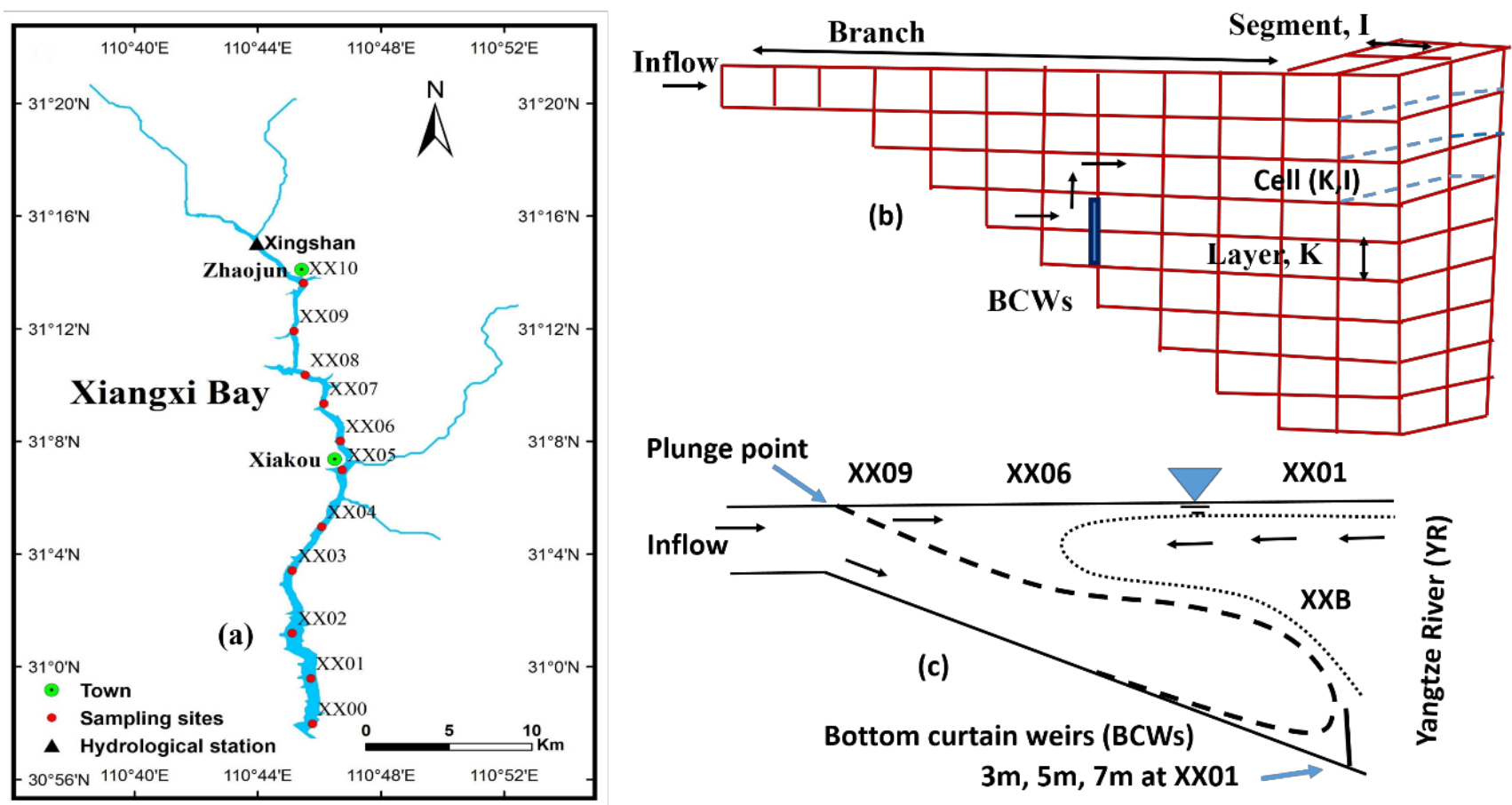

Figure 1. (a) Location of the sampling sites in XXB, (b) Schematic representation of bottom curtain weirs and (c) Illustration of BCWs in XXB 


\subsection{Bottom Curtain Weirs Mechanism}

BCWs were installed at three different sites in $\mathrm{XXB}$, XX09, XX06 and XX01 with corresponding heights of 3 $\mathrm{m}, 5 \mathrm{~m}$ and $7 \mathrm{~m}$ respectively as shown in Figure 1 (c). The BCWs effectively act as a barrier to flow and diffusion of heat across the width of the water body $[9,18]$. Inflow forms plunge flow before the BCWs and later travels as an underflow over the BCWs (Figure 1(c)). In addition, the mixing depth exceeds the critical depth [19]. When inflow or intrusive flow passes over the BCWs as underflow, mutual forces are exerted between the underflow and BCWs [20,21]. The underflow exerts forces on the BCWs $[22,23,24]$, and simultaneously BCWs must exert an equal and opposite force on the underflow or ambient water. As a result, eddies will be formed, which produce heat and have other effects on the thermal structure at XXB.

\section{Results}

\subsection{Effect of Bottom Curtain Weirs on Hydrodynamics in XXB}

BCWs were installed at heights of $3 \mathrm{~m}, 5 \mathrm{~m}$ and $7 \mathrm{~m}$ at three parts of $\mathrm{XXB}$, i.e., upstream, midstream and downstream corresponding to XX09, XX06 and XX01, respectively (Figure 2). The effects of the BCWs altered velocities significantly, as shown in Figure 2. The upstream and midstream velocities varied more than the downstream velocity because the downslope bottom density currents are blocked by the BCWs with height of $3 \mathrm{~m}, 5 \mathrm{~m}$ and $7 \mathrm{~m}$ at XX09 and XX06 (Figure 2).
Simultaneously, the intrusive density currents of mainstream YR of TGR were barricaded by the downstream BCWs with heights of $3 \mathrm{~m}, 5 \mathrm{~m}$ and $7 \mathrm{~m}$ at XX01 of XXB. However, because the downstream water level was deeper, the $7 \mathrm{~m}$ BCWs had less of an effects than the upstream and midstream BCWs as shown in Figure 2. The hydrodynamic conditions in XXB of TGR were much different during winter and autumn. The water level of TGR remained at the normal level of $175 \mathrm{~m}$ during winter and the flood control level of $145 \mathrm{~m}$ during summer. The water level daily fluctuations (WLDFs) were approximately $-2 \mathrm{~m}$ to $4 \mathrm{~m}$ in 2010 [2]. The BCWs enhanced the stirring of ambient water and increased the mixing depth along the water column in XXB as shown in the different scenarios in Figure 2. The underflows were susceptible to interaction with the BCWs and diverted their propagation path. The BCWs generated local turbulence that affected hydrodynamic properties such as velocities, inflow (from upstream) and intrusive flows (from mainstream YR), shear stress, buoyancy forces and acceleration due to gravity. The BCWs enhanced vertical mixing because density differences throughout the water column weaken vertical mixing. On the one hand, the BCWs obstructed underflow, but on the other hand, thermal stratifications broke in XXB as illustrated in Figure 2. The effects of BCWs, which can be employed for destratification, recirculation and artificial mixing, in XXB water are shown in Figure 2. Destratification and artificial mixing may then be an effective means of controlling algal blooms in XXB. Overall, the hydrodynamics were interrupted by the application of BCWs with heights of $3 \mathrm{~m}, 5 \mathrm{~m}$ and $7 \mathrm{~m}$ at XX09, XX06 and XX01 of XXB (Figure 2).

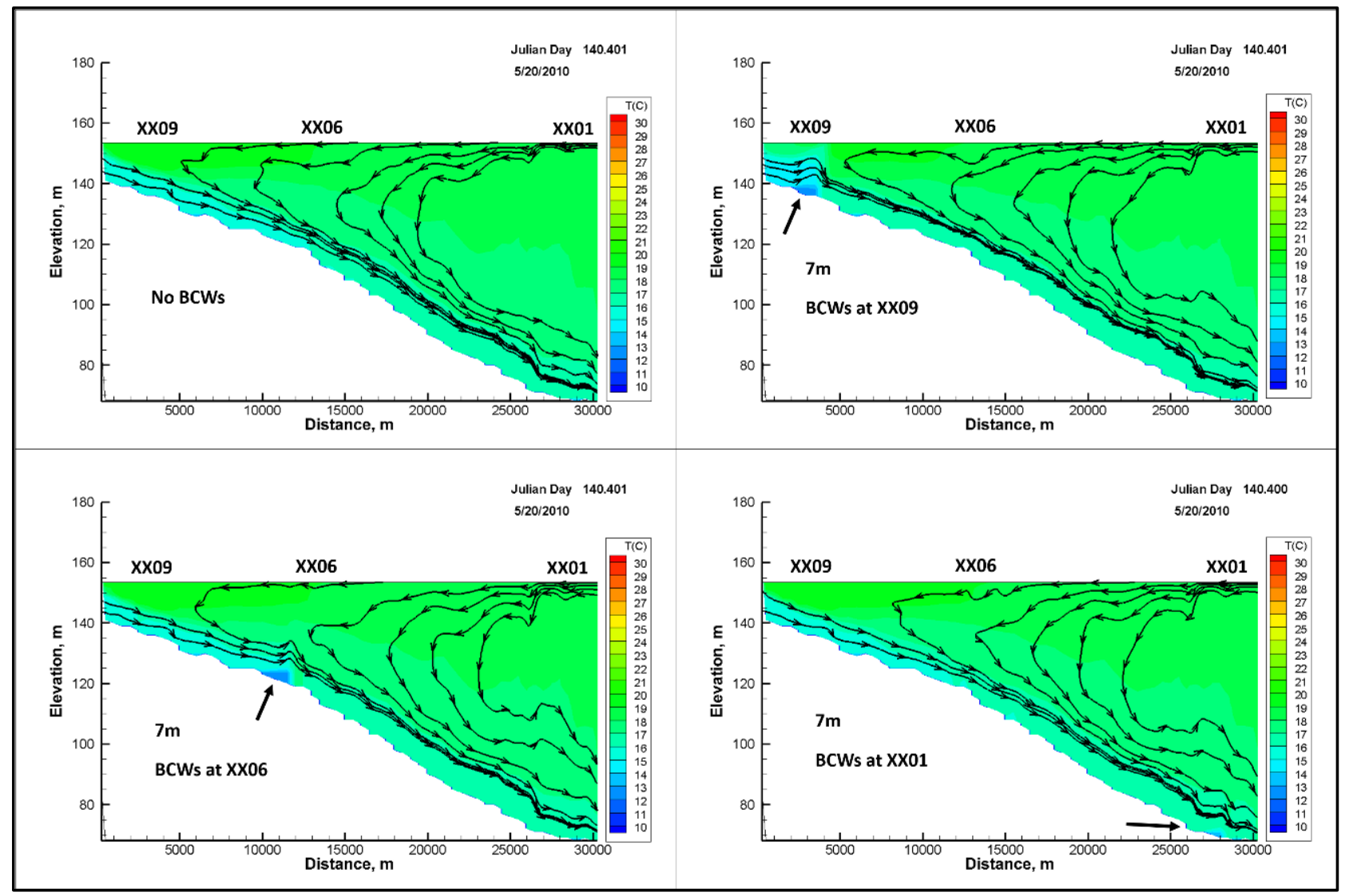

Figure 2. Spatial effects of the BCWs on hydrodynamic in XXB 


\subsection{Effects of Bottom Curtain Weirs on Temperature in XXB}

Density currents move under the influence of density differences among the three strata, i.e., epilimnion, metalimnion and hypolimnion and thermal stratification occurs in XXB. Thermal stratification is caused by density currents, which are effectively controlled by the BCWs in XXB. Normally, the onset of stratification occurs in early spring, and a thermocline exists in XXB. The most stable stratification conditions exist during the summer, when XXB exhibits large thermal gradients. As a result, the water quality of XXB may decrease because of algal blooms. The temporal effect of BCWs on temperature in $\mathrm{XXB}$ are shown in Figure 3.The thermal structure was broken due to the effect of the BCWs. Normally, temperatures increased around the BCWs because of the physical interaction between the BCWs and the density currents. In other cases, the temperatures decreased due to cold inflows from upstream and as an underflows and bottom intrusive flows from the mainstream YR in TGR as shown in Figure 3. The temperature differences (TDf) ranged from $>-0.5^{\circ} \mathrm{C}$ and $<0.5^{\circ} \mathrm{C}$ between no BCWs and BCWs during the whole simulation period in 2010. The simulated results showed that the TDf between no BCWs and BCWs at $3 \mathrm{~m}$ was $0.5{ }^{\circ} \mathrm{C}$ to $8{ }^{\circ} \mathrm{C}$ at XX09 from May 23 to September 06.
The maximum temperature decrease was $8.77^{\circ} \mathrm{C}$ to $9.22{ }^{\circ} \mathrm{C}$ on August 22-23 for BCWs with heights of $3 \mathrm{~m}$, $5 \mathrm{~m}$ and $7 \mathrm{~m}$ height at XX09 of XXB (Figure 3). The temperatures decreased from $1.05{ }^{\circ} \mathrm{C}$ to $1.63{ }^{\circ} \mathrm{C}$ on May 28, and June 16 for BCWs with heights of $3 \mathrm{~m}, 5 \mathrm{~m}$ and 7 $\mathrm{m}$ at XX06 (Figure 3). There was no distinguishable TDf between no BCWs and BCWs at $3 \mathrm{~m}, 5 \mathrm{~m}$ and $7 \mathrm{~m}$ at $\mathrm{XX} 01$ of $\mathrm{XXB}$ in the considered TDf range of $>-0.5^{\circ} \mathrm{C}$ and $<0.5^{\circ} \mathrm{C}$ (Figure 3 ). The BCWs not only reduced the temperature but also increased the temperature by approximately $0.63^{\circ} \mathrm{C}$ to $0.74^{\circ} \mathrm{C}$ on July 16 and September 22 for $3 \mathrm{~m}, 5 \mathrm{~m}$ and $7 \mathrm{~m}$ BCWs at XX09, as shown in Figure 3. The TDf between no BCWs and BCWs of $0.51^{\circ} \mathrm{C}$ to $8.8^{\circ} \mathrm{C}$ were observed on May 11 to September 8 for $3 \mathrm{~m}, 5 \mathrm{~m}$ and $7 \mathrm{~m}$ BCWs at XX09 of XXB as illustrated in Figure 3. There was no distinguishable TDf between no BCWs and BCWs for $3 \mathrm{~m}$, $5 \mathrm{~m}$ and $7 \mathrm{~m} \mathrm{BCWs}$ at XX01of XXB, as illustrated in Figure 3. Due to the effects of the BCWs of different heights, seasonally temperatures decreased in spring to autumn upstream of $\mathrm{XXB}$, as shown in Figure 3. The BCWs were produced distinct destratification throughout the whole simulation periods. As a result, the thermal structure was destroyed, and algal blooms occurred at much greater depths in XXB. When density currents entered from YR to XXB through hypolimnion strata as underflows, all of density currents were interrupted by the BCWs with heights of $3 \mathrm{~m}, 5 \mathrm{~m}$ and $7 \mathrm{~m}$.
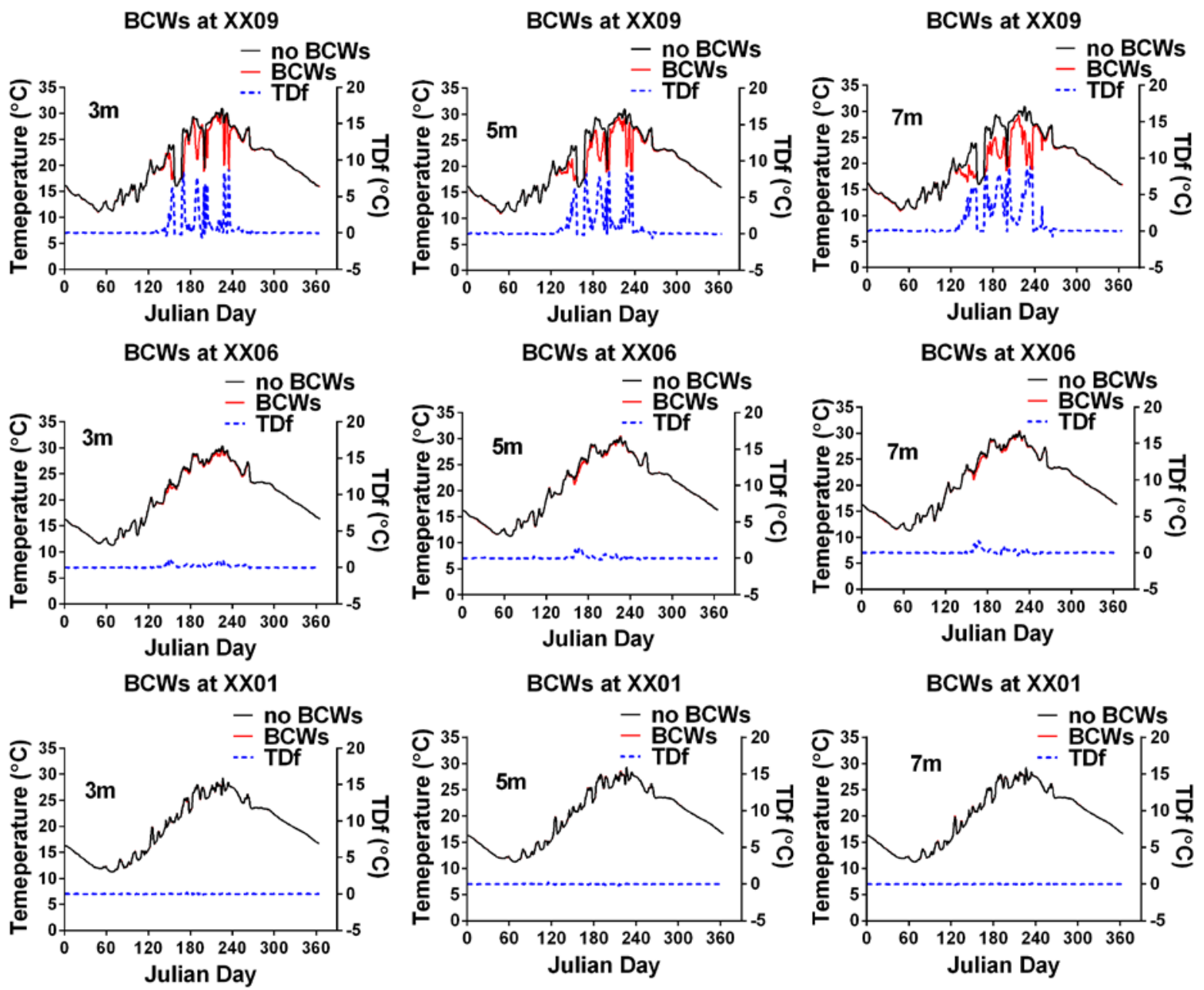

Figure 3. Temporal effects of BCWs on the thermal structure in XXB 


\subsection{Effects of BCWs on Chlorophyll-a Concentration in XXB}

The temporal effects of the BCWs on chlorophyll-a concentrations in XXB are shown in Figure 4. The chlorophyll-a concentrations reduction rates varied spatially and temporally. Time series of the simulated chlorophyll-a concentrations with no BCWs and BCWs with heights $3 \mathrm{~m}, 5 \mathrm{~m}$ and $7 \mathrm{~m}$ are shown in Figure 4, including chlorophyll-a concentrations differences (CDf) between no BCWs and BCWs. The chlorophyll-a concentrations were significantly reduced due to the effect of segment 9 , 25 and 58 BCWs corresponding to XX09, XX06 and $\mathrm{XX01}$, respectively. The upstream BCWs were more effective than the midstream and downstream BCWs. In summer (June, July and August), the inflow is cooler and submerges to the bottom, whereas warm water floats on the surface and creates a density difference between the epilimnion and hypolimnion strata. In addition, the upstream water level is shallower, and the inflow intensity is stronger, as a result, the inflow is more interrupted by the BCWs with heights of $3 \mathrm{~m}, 5 \mathrm{~m}$ and $7 \mathrm{~m}$. Moreover, thermal stratification is broken due to the effects of the BCWs with heights of $3 \mathrm{~m}, 5 \mathrm{~m}$ and $7 \mathrm{~m}$, as are the algal blooms underneath. Chlorophyll-a concentrations decreased dramatically at XX06 due to the effects of the BCWs. In the midstream, the $7 \mathrm{~m}$ and $5 \mathrm{~m} \mathrm{BCWs}$ are more effective than the $3 \mathrm{~m}$ BCWs because the water level is relatively higher than it is upstream (Figure 4).

The efficiency of the BCWs is lower at XX01 than at XX06 and XX09 because of the deep water downstream. As a result, BCWs with heights of 3-7 $\mathrm{m}$ are not effective enough at XX01 of XXB. Algal bloom reductions of greater than $30 \%$ were observed based on chlorophyll-a concentrations for no BCWs and BCWs. In the winter season (December, January and February), the observed chlorophyll-a concentrations were below $30 \%$, as a result, the winter season was not considered. Chlorophyll-a concentrations in XXB of more than 30\% were observed in spring (March, April and May), summer (June, July and August) and autumn (September, October and November) (Figure 4). CDfs were indicated by the broken line between no BCWs and BCWs as shown in Figure 4. CDf increased at XX09 and XX06 but decreased at XX01, as illustrated in Figure 4. The CDf is proportional to BCW performance. As CDf is increased, the BCW performance increases. The CDf between no BCWs and BCWs decreased significantly at XX01; as a result, chlorophyll-a concentration reduction rate was very low. In addition, the downstream intrusive density currents were thicker than those upstream; as a result, the performance of the 3 to $7 \mathrm{~m}$ BCWs was not significant. The chlorophyll-a concentrations reduction rates were lower in some scenarios because of storm inflow from upstream. Most of the inflow was blocked by the BCWs within a short time, and chlorophyll-a accumulated near the BCW area. At the midstream sampling site XX06, high chlorophyll-a concentrations of approximately $154 \mathrm{mg} / \mathrm{m}^{3}$ were observed as a result, density currents from both upstream and downstream interacted at this site. The BCWs produced chlorophyll-a concentration reductions of more than $44 \%, 28 \%$ and $4 \%$ at XX09, XX06, and XX01, respectively, in $\mathrm{XXB}$, as shown in Figure 4. The $3 \mathrm{~m}$ BCWs showed performance of more than 33\%, compared $27 \%$ and $16 \%$ for the $7 \mathrm{~m}$ and $5 \mathrm{~m}$ BCWs, respectively, as shown in Figure 4 and Table 1.
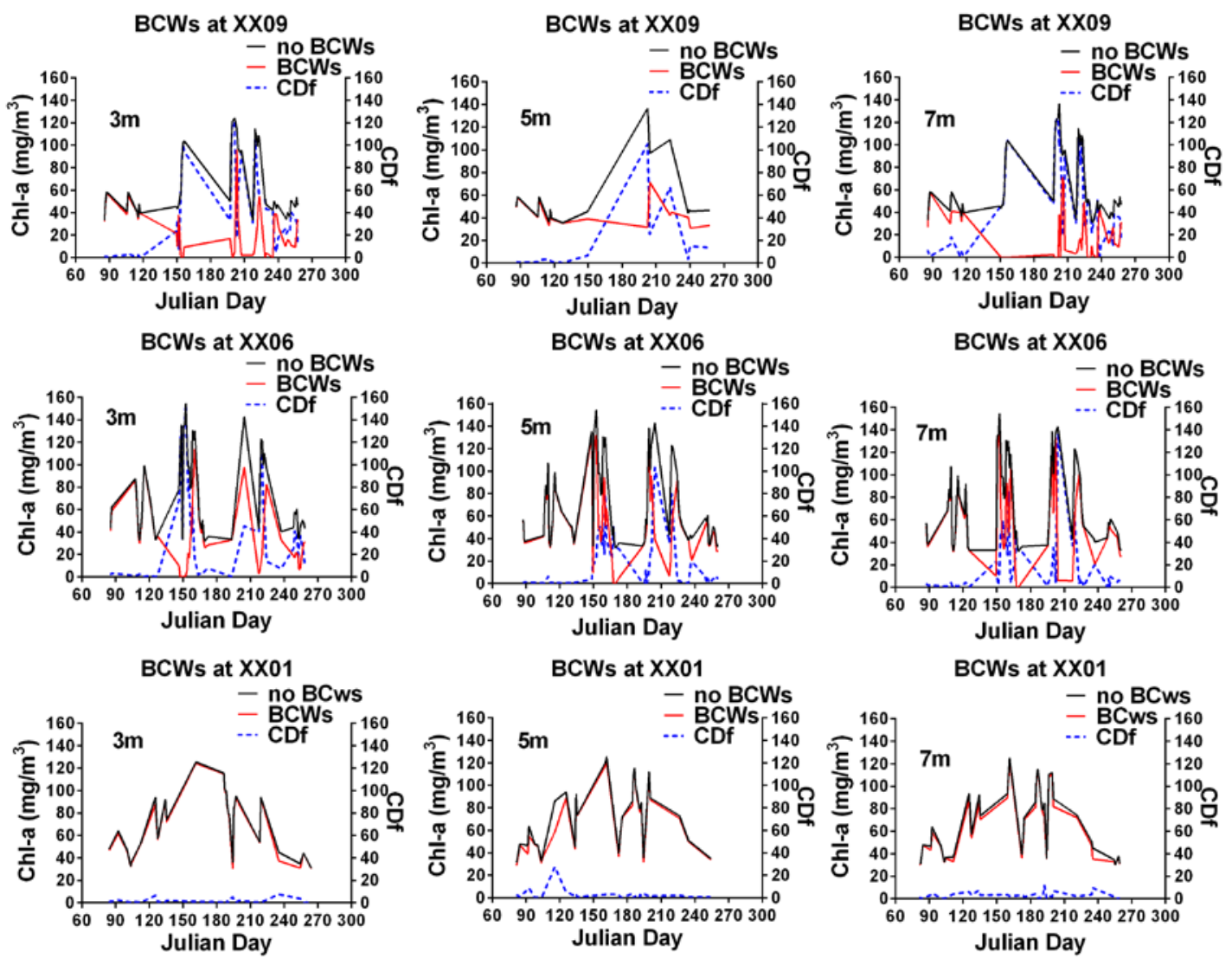

Figure 4. Temporal effects of BCWs on Chl-a in XXB of TGR 
Table 1. BCWs effectiveness scenarios at different locations of XXB in 2010

\begin{tabular}{|c|c|c|c|c|c|c|c|}
\hline \multirow{2}{*}{$\begin{array}{c}\mathrm{XXB} \\
\text { locations }\end{array}$} & \multirow{2}{*}{$\begin{array}{l}\text { BCW } \\
\text { height }\end{array}$} & \multirow[t]{2}{*}{ Scenarios } & \multicolumn{3}{|c|}{$\begin{array}{c}\text { BCW seasonal Chl-a reduction efficiency } \\
(\%)\end{array}$} & \multirow{2}{*}{$\begin{array}{l}\text { Yearly efficiency (\%) per } \\
\text { scenario }\end{array}$} & \multirow{2}{*}{$\begin{array}{c}\text { Average efficiency } \\
\text { (\%) }\end{array}$} \\
\hline & & & Spring & Summer & Autumn & & \\
\hline \multirow{4}{*}{ XX09 } & $3 \mathrm{~m}$ & Seg9 & 15 & 75 & 62 & 51 & \multirow{4}{*}{44} \\
\hline & $5 \mathrm{~m}$ & Seg9 & 5 & 41 & 29 & 25 & \\
\hline & $7 \mathrm{~m}$ & Seg9 & 34 & 76 & 57 & 56 & \\
\hline & $3 \mathrm{~m}$ & Seg25 & 34 & 43 & 59 & 45 & \\
\hline \multirow[t]{3}{*}{ XX06 } & $5 \mathrm{~m}$ & Seg25 & 6.3 & 38.43 & 9 & 18 & \multirow[t]{2}{*}{28} \\
\hline & $7 \mathrm{~m}$ & Seg25 & 6 & 44 & 11 & 20 & \\
\hline & $3 \mathrm{~m}$ & Seg58 & 3 & 3.35 & 4 & 3.45 & \\
\hline \multirow[t]{3}{*}{ XX01 } & $5 \mathrm{~m}$ & Seg58 & 7.51 & 3 & 3 & 4.5 & \multirow[t]{3}{*}{4} \\
\hline & $7 \mathrm{~m}$ & Seg58 & 5 & 5 & 2 & 4 & \\
\hline & $\mathrm{Ave}^{*}$ & & $13 \%$ & $37 \%$ & $26 \%$ & & \\
\hline
\end{tabular}

${ }^{1.1}$ Yearly efficiency (\%) per scenario means each segment and corresponding BCW height.

${ }^{1.2}$ Average efficiency (\%) means average algal blooms reduction percentage for XX09, XX06 and XX01, respectively.

${ }^{1.3} \mathrm{Ave}^{*}$ means seasonal algal bloom reduction percentage at BCWs installed sites.

\section{Effectiveness of Bottom Curtain Weirs (BCWs)}

\subsection{BCWs Efficiency}

The effectiveness of the BCWs was determined by using the normalized curtain efficiency $\left(B C_{e f f}\right)$ which is defined in equation (1).

$$
B C_{e f f}=\frac{B C_{\text {no BCWs }}-B C_{B C W s}}{B C_{\text {no BCWs }}} * 100 \% .
$$

The efficiency of the BCWs varied at the different locations such as XX09, XX06 and XX01 in XXB of TGR (Table 1). The model also predicted overall BCW efficiency of $44 \%, 28 \%$ and $4 \%$ at XX09, XX06 and XX01, respectively, as shown in Figure 4 and Table 1. Seasonal algal bloom reduction rates of more than $37 \%$, $26 \%$ and $13 \%$ were observed in summer, autumn and spring respectively, in 2010 as shown in Figure 4 and Table 1 . The BCW performance was more than 33\%, 27\% and $16 \%$ for heights of $3 \mathrm{~m}, 7 \mathrm{~m}$ and $5 \mathrm{~m}$ respectively (Table 1). In winter, algal blooms occurred in XXB no less than $30 \%$ of days, and thus winter was not considered in this research.

\section{Discussion}

\subsection{Effect of BCWs on Hydrodynamics in XXB of TGR}

The proposed BCWs were simulated using a two-dimensional, laterally averaged, hydrodynamic model based on CE-QUAL-W2. The CE-QUAL-W2 model is appropriate for XXB of TGR [2]. BCWs have been used with the CE-QUAL-W2 model to control algal blooms in reservoirs such as the Daecheong Dam Reservoir, in Korea, and Terauchi Dam Reservoir, in Japan [9-12,14,25]. BCWs were installed at three different sites, i.e., upstream, midstream and downstream, corresponding to XX09, XX06 and XX01, respectively, in XXB. The water level was shallower at the upstream site compared with midstream and downstream. Most of the underflow was interrupted by the BCWs with heights of $3 \mathrm{~m}, 5 \mathrm{~m}$ and 7 $\mathrm{m}$; as a result, the chlorophyll-a concentrations reduction rate increased upstream in XXB (Figure 2). Inflowing cooler water resulted in density differences and underflow, which were interrupted by the BCWs with heights of $3 \mathrm{~m}$, $5 \mathrm{~m}$ and $7 \mathrm{~m}$. The algal blooms filtered upstream to the immediate downstream of the reservoir (Figure 4). As a result, the chlorophyll-a reduction rates were higher in segment 9 than in segments 25 and 58 (Table 1). Bottom intrusion also occurred in XXB as an underflow from the mainstream YR of TGR. All of these underflow were interrupted by the BCWs of different heights, but all the deeper water levels downstream, the performance of the BCWs with heights of $3 \mathrm{~m}$ to $7 \mathrm{~m}$ were lower compared with the performances upstream and midstream. The BCWs with different heights obstructed underflow density currents at different locations in XXB. Due to the effects of the BCWs, large amounts of kinetic energy were produced continuously through the whole process and as a result, the hypolimnion strata temperatures fluctuated. The BCWs enhanced eddy diffusivity at XX09, XX06 and XX01 of XXB. Eddy diffusivity is a bidirectional process with continuous transportation. Temperature fluctuations are occurred due to the effect of the BCWs. Thermal stratification is responsible for density differences in XXB $[1,2,4]$. In $\mathrm{XXB}$, the metalimnion strata is very thin, and epilimnion algal blooms are transported very quickly to the hypolimnion due to the effect of the BCWs. Inflow density currents pass as an underflow over the BCWs; as a result, mutual forces are exerted between the underflows and BCWs. The underflow exerted a force on the BCWs, and simultaneously the BCWs must exert an equal and opposite force on the underflow and ambient water around the BCWs. Thermal stratification occurs naturally from the onset of spring to autumn turnover in XXB [2]. After installation of the BCWs, the simulation results showed that the stratification patterns changed and that destratification occurred in XXB. The destratification was well-developed on June 20, 2010.

\subsection{Effects of BCWs on Water Quality of XXB of TGR}

In this study, BCWs were used to control algal blooms in XXB through the CE-QUAL-W2 model. In previous study, floating curtain weirs were used to control algal 
blooms in different reservoirs [9-11,14,25]. Simulation results indicated that the algal blooms reduction efficiency of the BCWs was more than $44 \%, 28 \%$ and $4 \%$ upstream, midstream and downstream, corresponding to XX09, XX06 and XX01, respectively as shown in Figure 4 and Table 1. The observed effectiveness of the BCWs was 13 to $37 \%$ in spring, summer and autumn, with a maximum efficiency of approximately $57 \%$ in summer, as shown in Figure 4 and Table 1. The activity of the BCWs persisted longer upstream and midstream, corresponding to XX09 and XX06, than downstream at XX01, with values of 6143\% (Table 1). The interruption of upstream interflow by the BCWs was greater than that of the downstream underflow. As a result, the efficiency of the BCWs was lower downstream than upstream. The effectiveness of the BCWs increased at segments 9 and 25 corresponding to XX09 and XX06 instead of segment 58 corresponding to XX01 in spring, summer and autumn. During May 27-31, June 1-4, June 16-18, July 18, July 22, August 16-18 and August 23-24, the $3 \mathrm{~m}, 5 \mathrm{~m}$ and $7 \mathrm{~m} \mathrm{BCW}$ reduced algal blooms by up to $99 \%$ at XX09 and XX06 of XXB. Density currents enter from upstream and downstream over the surface, middle and bottom as overflow, interflow and underflow. The BCWs interrupted all of these density currents and controlled algal blooms in XXB. Lon-term field monitoring data recorded that algal blooms occur more in the spring, summer and autumn in XXB [1,2,15]. Algal blooms can occur rapidly within a couple of days when the reservoir is characterized by abundant resources such as nutrients, a sufficient water temperature, available solar radiations, and a lack of perturbation or disturbances. According to the Intermediate Disturbances Hypothesis (IDH) algal blooms occur more with intermediate disturbances [26,27]. Algal blooms rapidly increase with the ratio of euphotic depth to mixing depth during the spring and summer. If the value of the ratio decreases because the mixing depth rapidly increases and exceeds the euphotic depth, algal blooms disappear rapidly because they become mixed much deeper according to the critical depth theory [19]. The performance of the BCWs should destroy the thermal stratification and rapidly increase the mixing depth to exceed the euphotic depth and move the algal blooms underneath.

\section{Conclusions}

The installation of BCWs upstream, midstream and downstream in a subtropical reservoir of XXB in China, corresponding to sites XX09, XX06 and XX01, respectively, was proposed for the control algal blooms. A laterally averaged two-dimensional hydrodynamic and water quality model (CE-QUAL-W2) was used to simulate hydrodynamics, temperature and chlorophyll-a concentration using data collected in XXB from January to December 2010. Underflow density currents are the environmental issue that influences algal bloom all year round in XXB. Underflow density currents transport nutrients, solids and other substances both sides upstream and downstream and stores these materials in the midstream of XXB. Algal blooms occur severely in the midstream simultaneously upstream and downstream of XXB. Maximum chlorophyll-a concentration of 154 $\mathrm{mg} / \mathrm{m}^{3}$ were observed in the scenario with no BCWs at XX09 and XX06. The performance of the BCWs suggests that overall chlorophyll-a concentrations were markedly reduced by $4-44 \%$ as a function of $\mathrm{BCW}$ height and location. Seasonal algal bloom reduction rates of more than $37 \%, 26 \%$ and $13 \%$ were observed in summer, autumn and spring respectively. During May 27-31, June 2-4, June 16-18, August 16-18 and August 23-24, the $3 \mathrm{~m}$, $5 \mathrm{~m}$ and $7 \mathrm{~m}$ BCWs reduced algal blooms by up to $99 \%$ at XX09 and XX06 of XXB. When density currents enter from upstream and downstream over the surface, middle and bottom as overflow, interflow and underflow, all of these density currents are blocked by the BCWs. The BCW performance according to height was more than $33 \%, 27 \%$ and $16 \%$ for $3 \mathrm{~m}, 7 \mathrm{~m}$ and $5 \mathrm{~m}$ respectively. The BCWs were used to control algal blooms in XXB of the subtropical reservoir of China through the CE-QUAL-W2 model. Numerical models of BCWs are much more attractive, much less expensive and time saving for controlling algal blooms in subtropical reservoirs.

\section{Acknowledgments}

The authors are grateful to all of the members of the Hubei Key Laboratory of Ecological Restoration of Riverlakes and Algal Utilization, Wuhan, China and China Three Gorges University (CTGU), College of Hydraulic \& Environmental Engineering, Yichang, 443002, Hubei, China for participating in the field monitoring.

\section{Funding}

This research was supported financially by the National Key R\&D Program of China (Grant No. 2016YFC0401702), National Natural Science Foundation of China (Grant Nos. 91647207, 51709096) and China Scholarship Council (CSC) (File No. 2014DFHC97).

\section{References}

[1] Ji, D., et al., Impacts of water level rise on algal bloom prevention in the tributary of Three Gorges Reservoir, China. Ecological Engineering, 2017. 98(Supplement C): p. 70-81.

[2] Ma, J., et al., Modeling density currents in a typical tributary of the Three Gorges Reservoir, China. Ecological Modelling, 2015. 296(Supplement C): p. 113-125.

[3] Yang Z J, L.D.F., Ji D B, , Influence of the impounding process of the Three Gorges Reservoir up to water level $172.5 \mathrm{~m}$ on water eutrophication in the Xiangxi Bay. Science China Technological Sciences, 2010. 53: p. 1114-1125.

[4] Yang, Z., et al., An eco-environmental friendly operation: An effective method to mitigate the harmful blooms in the tributary bays of Three Gorges Reservoir. Science China Technological Sciences, 2013. 56(6): p. 1458-1470.

[5] Benjamin, T.B., Gravity currents and related phenomena. Journal of Fluid Mechanics, 1968. 31(2): p. 209-248.

[6] Ashida, K. and S. Egashira, Basic study on turbidity currents. Vol. 1975. 1975. 37-50.

[7] Kao, T.W., Density Currents and Their Applications Journal of the Hydraulics Division, 1977. 103 (5): p. 543-555.

[8] R. L. Doneker; J. D. Nash; and G. H. Jirka, Pollutant Transport and Mixing Zone Simulation of Sediment Density Currents. Journal of Hydraulic Engineering 2004. 130(4): p. 349-359. 
[9] Asaeda, T., et al., A new technique for controlling algal blooms in the withdrawal zone of reservoirs using vertical curtains. Ecological Engineering, 1996. 7(2): p. 95-104.

[10] Imteaz, M.A. and T. Asaeda, Artificial mixing of lake water by bubble plume and effects of bubbling operations on algal bloom. Water Research, 2000. 34(6): p. 1919-1929.

[11] Asaeda, T., et al., Control of algal blooms in reservoirs with a curtain: a numerical analysis. Ecological Engineering, 2001. 16(3): p. 395-404.

[12] H. S. Lee, S.W.C., J. K. Choi \& B. H. Min, Feasibility of curtain weir installation for water quality management in Daecheong Reservoir. Desalination and Water Treatment 2010. 19: p. 164-172.

[13] Priyantha, D.G.N., et al., Modelling effects of curtain method on algal blooming in reservoirs. Ecological Modelling, 1997. 98(2): p. 89-104.

[14] Se-Woong Chung, H.L.a.Y.J., The effect of hydrodynamic flow regimes on the algal bloom in a monomictic reservoir. Water Science \& Technology 2008. 58(6): p. 1291-8.

[15] Yang, Z., et al., An eco-environmental friendly operation: An effective method to mitigate the harmful blooms in the tributary bays of Three Gorges Reservoir. Science China Technological Sciences, 2009. 56(6): p. 1458-1470.

[16] Berger, C.J., Wells, S.A.,, Modeling the effects of macrophytes on hydrody- namics. J. Environ. Eng., 2008. 134 (9): p. 778-788.

[17] Wells, S., Wells, V., Berger, C., Impact of Phosphorus Loading from the Watershed on Water Quality Dynamics in Lake Tenkiller. ASCE, 2012: p. 888-899.

[18] Dias, F. and J.-M. Vanden-Broeck, Open channel flows with submerged obstructions. Journal of Fluid Mechanics, 2006. 206(-1).

[19] Sverdrup, H.U., On conditions for the vernal blooming of phytoplankton. ICES Journal of Marine Science, 1953. 18(3): p. 287-295.

[20] Helfer, F., H. Zhang, and C. Lemckert, Modelling of lake mixing induced by air-bubble plumes and the effects on evaporation. Journal of Hydrology, 2011. 406(3): p. 182-198.

[21] Sambuco, E. and J.A. Whitehead, Hydraulic control by a wide weir in a rotating fluid. Journal of Fluid Mechanics, 2006. 73(03).

[22] Akiyama, J., and Stefan, H. G., Theory of Plunging Flow into a Reservoir. ASCE, 1984. 110(4): p. 484-499.

[23] Akiyama Juichiro, J.A.K., Yamasaki Tsutomu, Flow Characteristics of Surface Currents and Mixing by Curtains. Doboku Gakkai Ronbunshuu 2002. B 46: p. 1025-1030.

[24] Akiyama Juichiro, J.A.K., YAMASHITA Naoki, URA Masaru, Control of Plunge Flows in Reservoir by Curtains for Countering Freshwater Red-Tide Blooms. Doboku Gakkai Ronbunshuu 2002. 46: p. 1013-1018.

[25] Chung, S.W.L., H. Jung, Y., The effect of hydrodynamic flow regimes on the algal bloom in a monomictic reservoir. Water Sci Technol, 2008. 58(6): p. 1291-8.

[26] Reynolds, C.S., The intermediate disturbance hypothesis and its applicability to planktonic communities: Comments on the view of Padisak and Wilson. New Zealand Journal of Ecology 1995. 19(2)

[27] Connell, J.H.a.R.O.S., Mechanisms of succession in natural communities and their role in community stability and organization. The American Naturalist 1977. 111(982): p. 1119-1144.

(C) The Author(s) 2019. This article is an open access article distributed under the terms and conditions of the Creative Commons Attribution (CC BY) license (http://creativecommons.org/licenses/by/4.0/). 